\title{
An Novel Image Recognition method based on Three-way Decision
}

\author{
Jin $\mathrm{Dai}^{1}$, Shuai Shao ${ }^{1}, \mathrm{Zu} \mathrm{Wang}^{1}$ and Xianjing Zhao ${ }^{1}$ \\ ${ }^{1}$ School of Software Engineering, Chongqing University of Posts and Telecommunications
}

\begin{abstract}
The traditional recognition method takes the low-level information of the image as the foundation. The image recognition center of gravity is biased towards the typical features, and achieves the effect of recognition by region-dependent segmentation. Because the general image segmentation is a regular rectangle, easily lead to the same target is divided into different sub-blocks, ignoring the image of the fuzzy part, so the image recognition is not complete. An image recognition algorithm based on threeway decision is proposed. It takes full advantage of effective information in the image, improving the image recognition accuracy. First, this method divided the image into three regions: positive region, negative region and delay decision region. Second, an iterative process is performed on the region of the delay decision. Final, image recognition is performed on the positive sample region. Based on the basic theory of the three-way decision, the more obvious the decision result is, the more iterations are, and the information is added to the classifier until the blurred part of image cannot be divided. Finally, to achieve the realize effective image recognition. This method simulates the process of human cognition effectively, and makes the utilization of the effective information reach the maximum in the recognition process. The results of the experimental analysis showed that the method is more concise and efficient, and the recognition accuracy is more accurate.
\end{abstract}

\section{INTRODUCTION}

Image recognition refers to the use of computers to image processing, analysis and understanding to identify a variety of different models of the goals and the objects of the technology. Based on content of image recognition is a very active research field since the 1970 s, its driving force is derived from two major research directions: database system and computer vision [1]. At present, the initial image recognition research focused on how to select the appropriate global features to describe the image content and what kind of similarity measure for image matching. Owing to the immaturity of computer vision and artificial intelligence, the further development of image recognition is affected. Because of the global image features, this method is only suitable for simple images or background images.

In recent years, based on image recognition related feedback technology has also become a research hotpot. At present, the computer vision and pattern recognition technology cannot achieve the object of high efficiency recognition, which makes it difficult to describe the semantic and abstract layer semantics of object layer. Therefore, the study of image semantic features is focused on the first layer of semantic feature mode [2].
Because the simple semantic feature extraction and analysis is based on the use of complex semantic information, and the computer's understanding of the image content generally refers to the image of the lowlevel visual features, such as color, texture, shape, etc... In fact, the human understanding of the content of the image far more than the visual features of the image. There is a large gap between the low-level and the highlevel semantic features of the image, called the "semantic gap" $[2,3]$.

In summary, the general image recognition methods are based on the image of the low-level semantics to study. Due to the computer's performance, can only be programmed to perform a single instruction, resulting in the general method of image recognition rate is low. How to improve the image recognition rate and understand the deep semantics of the image becomes the research direction which needs to be solved urgently.

At present, three-way decision theory is applied to solve complex problems and computer simulation of human thinking methods. Many scholars combined the three-way decision and particle computing in the study, and most scholars focused on image recognition research about describe the image content (low-level information), and it is still less that integrating the classifier to improve the classification performance of the method. Due to the complexity content of the image (high-level semantics),

*Corresponding author: \{ Jin Dai, Shuai Shao \} daijin@cqupt.edu.cn, nandehutubai@hotmail.com 
the image classification task is very difficult, and the three-way decision theory is generally used for fuzzy information, which can effectively classify the fuzzy information in the image in order to achieve the purpose of improving the image recognition rate. In this paper, we purposed an image recognition algorithm based on the three-way decision, and we improved the image classifier algorithm.

\section{RELATED WORKS}

Image recognition is to classify the processed image. First, the category name is determined. Then, the feature to be extracted is selected on the basis of the segmentation, and some parameters are measured. Finally, the image is classified according to the measurement result. In order to better recognition the image, we need to analyse the whole structure of the image, describing the image, so that the main information of the image to get an explanation and understanding, and through many of the structural relationship to deepen understanding of the image. So, the image recognition is in each of the parts after the division, to find its shape and texture and other characteristics, that is feature extraction (sometimes including image segmentation), in order to classify the image, and structural analysis the entire image.

At present, the mainstream image recognition process includes pre-processing, image segmentation, image feature extraction, image classification.

The three-way decision theory is developed in recent years, a method of dealing with uncertainty decisionmaking, is a complex human cognitive "Trisecting and Acting" model [6]. The three-way decision theory is a decision-making model based on human cognition $[6,12]$. Which argues that people can make quick judgments immediately in the course of actual decision-making, for those who have a good grasp of acceptance or rejection. Immediate decision-making things, people tend to postpone the judgment of the incident, that is delay decision [6]. In the process of practical decision-making, there are the complexity of the decision-making environment, the incompleteness of the information acquisition, the inconsistency of the expert opinions in the group decision-making [7]. The uncertainty of the knowledge and the ambiguity of the thinking situation, the decision-maker in most cases it is difficult to accurately give the specific value of the loss function. In general, the meaning of uncertainty is broad, including randomness, ambiguity, imperfectness, instability and inconsistency [8]. The three-way decision is divided into two modes: the double evaluation function and the single evaluation function [12].

Definition 1: The three-way decision based on the double evaluation function [6]

Definition 2: The three-way decision based on the single evaluation function [6]

Axiom 1: The more knowledge, the three-way decision is clearer [13]

The more information is known, the more likely it is to accept and reject the decision. The less likely it is to promise, the smaller the known information, the less likely to accept and reject, and the greater the likelihood of no commitment. The more the features of the set are extracted, the more the same or opposite properties can be determined. The less the different attributes are, the more accurate the degree of association is. The less the feature extraction is, the less the same or the opposite the more attributes, the degree of contact is rougher.

\section{THE THREE-WAY DECISION IN IMAGE RECOGNITION}

To improve the accuracy of image recognition has always been a difficult problem in the field of image recognition. In general, the research of image recognition focuses on the low-level visual features of images, which leads to a single image recognition algorithm, and the researchers focused on the research in the low-level visual feature points. It is easy to result in the recognition of the useful information in the image is not complete, thus reducing the image recognition rate. In this paper, we proposed an image recognition method based on three-way decision, which makes useful information in the image to make the image recognition more accurate.

- The main problem of existence

The image segmentation of the rules causes the same objects to be split into different sub-blocks.

At present, most scholars focus on the classification of image recognition in the description of image content (the low-level information), such as color, texture, shape, etc... In fact, the human understanding of the image semantic information expressed much more the visual features. The existing image segmentation usually divides the image directly into a regular rectangle, causing the same objects to be divided into different image sub-blocks, resulting in errors in the classification. Existing methods of identification for improved fusion of classifiers in order to improve image recognition performance are still less. Due to the complexity of the image information, the image classification task is difficult, and the image semantic classification is still a challenging subject in the field of image recognition and computer vision.

- The three-way decision in image recognition In view of the above problems, this paper proposed an image recognition method based on three-way decision. The method uses three mechanisms to deal with fuzzy information. The uncertain regions of different subblocks in image segmentation will be judged again. Because this method divided the image by increasing the decision condition of the classifier, the images in different sub-blocks are divided into the same sub-blocks in the process of continuous iteration, which effectively avoids the problems in traditional method. Each of the three-way decision classifier to increase the conditions of the decision is from the existing conditions after the screening of information, so each increase in the information according to the current image of their own attributes, and constantly adjust the partition region, thus achieve accurate recognition purpose.

- Technological Scheme 
In this paper, we used efficient image segmentation method to improve the recognition rate. We used the multi-feature, segmentation, detection, multi-classifier and other methods, the depth of the sample image training, in reducing the image loss at the same time, splitting the target region, and retraining the new information. The composite SVM used in this paper is an improved version based on SVM. The final detection and recognition is realized by fusion and segmentation. Aiming at the problem of low recognition rate of general method, this paper proposed an image recognition method based on three-way decision. According to the characteristics of human cognitive things. Because of the unique decision-making characteristics of the three-way decision, the new decision information is added to the decision-making condition in the initial judgment condition. After completion, the positive and negative regions that have been divided will be trained as a new training set, and a new judgment condition will be added to the region of the delay decision until the region of the delay decision cannot be divided. Finally, the image recognition is performed. As stated in Axiom 1, each training will add new decision conditions to make the classification of the three-way decision classifiers is clearer, the delay decision-making part of the smaller and smaller, until a certain critical value, and finally to achieve the image recognition.

According to the above method, put forward the technical roadmap, as shown in Figure 2.

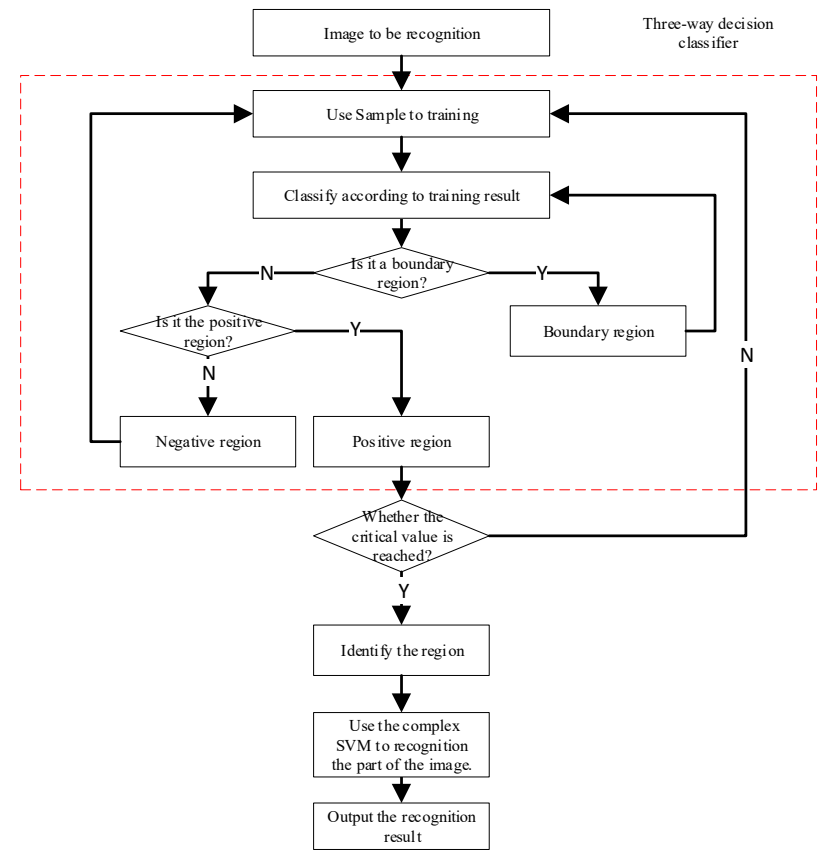

Figure 2: Technology Roadmap.

\section{IMAGE RECOGNITION ALGORITHMS BASED ON THREE-WAY DECISION}

Corresponding to the three-way decision, we use $\alpha, \beta$ and ${ }^{\xi}$, respectively, that acceptance, rejection and delay decision. Assuming that the evaluation function is defined as $P_{r}(X \mid[x])$, the risk function is $R(\Delta \mid x)$, where the decision action for $\mathrm{x}$ is $\Delta$. Based on the cost matrix given in Tab 1, the following states can be estimated as follows:

$$
\begin{aligned}
& \text { Acceptable: } R(\alpha \mid x)=\lambda_{\alpha p} \cdot P_{\beta}(X \mid[x])+\left(1-P_{\beta}(X \mid[x])\right) \\
& \text { Refuse: }{ }^{R(\beta \mid x)=\lambda_{\beta p} \cdot P_{\beta}(X \mid[x])+\left(1-P_{\beta}(X \mid[x])\right)} ; \\
& \text { Delay decision: } R(\xi \mid x)=\lambda_{\zeta p} \cdot P_{\beta}(X \mid[x])+\left(1-\mathrm{P}_{\beta}(X \mid[x])\right) .
\end{aligned}
$$

In the decision-making process, based on the risk function, select the risk of the smallest decision-making action: When the condition $R(\alpha \mid x) \leq R(r \mid x) \wedge R(\alpha \mid x) \leq R(n \mid x)$ is satisfied, the acceptance is selected; when the condition $R(r \mid x) \leq R(\alpha \mid x) \wedge R(r \mid x) \leq R(n \mid x)$ is satisfied, the rejection is selected; when the condition $R(n \mid x) \leq R(\alpha \mid x) \wedge R(n \mid x) \leq R(r \mid x)$ is satisfied, the delay decision is selected. Assumed that the risk function satisfies the following conditions:

$$
\begin{aligned}
& \lambda_{\alpha p} \leq \lambda_{\xi p}<\lambda_{\beta p}, \quad \lambda_{\beta n} \leq \lambda_{\xi n}<\lambda_{\alpha n}, \\
& \frac{\lambda_{\beta p}-\lambda_{\xi p}}{\lambda_{\xi n}-\lambda_{\beta n}}>\frac{\lambda_{\xi p}-\lambda_{\alpha p}}{\lambda_{\alpha n}-\lambda_{\xi n}}
\end{aligned}
$$

By substituting $R(\alpha \mid x), R(r \mid x)$ and $R(n \mid x)$ into the above three inequalities, the following results can be obtained:

If $P_{r}(X \mid[x]) \geq \alpha$, select accept; if $P_{r}(X \mid[x]) \leq \beta$, select reject; if $\beta \leq P_{r}(X \mid[x]) \leq \alpha$, select delay decision.

Table 1: Two Kinds of State Decision Problem Matrix.

\begin{tabular}{|l|l|l|}
\hline \multirow{2}{*}{ Decision action } & \multicolumn{2}{|l|}{ Objective state } \\
\cline { 2 - 3 } & Satisfaction: & Dissatisfaction: \\
\hline Acceptance: $\alpha$ & $\lambda_{\alpha p}$ & $\lambda_{\alpha n}$ \\
\hline Rejection: $\beta$ & $\lambda_{\beta p}$ & $\lambda_{\beta n}$ \\
\hline $\begin{array}{l}\text { Delay decision: } \\
\xi\end{array}$ & $\lambda_{\xi p}$ & $\lambda_{\xi n}$ \\
\hline
\end{tabular}

Based on the above theory, the following algorithm is proposed. Algorithm 1 is an image classifier algorithm based on three-way decision. we purposed to divide the image into three regions, and did the preliminary work for the recognition of algorithm 2. Algorithm 2 is a recognition algorithm based on three-way decision, through the algorithm 1, the image segmentation of the part of the recognition.

Algorithm 1: Image Classification Algorithm based on Three-way Decision

Input: The image dataset $\psi$.

Output: An image classifier $M$ based on three-way decision.

Begin

Step1: Input the image training dataset $\psi$.

Step2: According to the state condition in Table 1, classifier parameters $\lambda_{\alpha p}, \lambda_{\alpha n}, \lambda_{\beta p}, \lambda_{\beta n}, \lambda_{\xi p}, \lambda_{\xi n}$ are trained.

Step3: The positive sample region is obtained according to the value of formula: $\alpha=\frac{\lambda_{\alpha n}-\lambda_{\xi n}}{\left(\lambda_{\alpha n}-\lambda_{\xi n}\right)+\left(\lambda_{\xi p}-\lambda_{\alpha p}\right)}$, The negative sample region is 
obtained according to the value of formula: $\beta=\frac{\lambda_{\xi n}-\lambda_{\beta n}}{\left(\lambda_{\xi n}-\lambda_{\beta n}\right)+\left(\lambda_{\beta p}-\lambda_{\xi p}\right)}$

Step4: The delay decision region is obtained according to the value of $\mu$. and $\beta<\mu<\alpha$.

Step5: Output the image classifier $M$.

End

Through this algorithm, the image dataset is trained to get an available classifier based on three-way decision.

Algorithm 2: Image Recognition Algorithm based on Three-way Decision

Input: Image $\mathrm{C}$ to be recognized.

Output: The Image Recognition Result N.

Begin

Step1: Input the image C.

Step2: Using the classifier $M$ in algorithm 1, the image $\mathrm{C}$ is divided into three regions: positive region( $\mathrm{P})$, negative region( $\mathrm{N})$, delay decision region(B).

Step3: It is determined whether or not the delay decision region(B) has reached the recognition threshold value $\xi=\frac{\lambda_{\alpha n}^{\prime}-\lambda_{\xi n}^{\prime}}{\left(\lambda_{\alpha n}^{\prime}-\lambda_{\xi n}^{\prime}\right)+\left(\lambda_{\xi p}^{\prime}-\lambda_{\alpha p}^{\prime}\right)}$, and if not, repeat step 2 for the $\mathrm{B}$ region.

Step4: If the $\xi$ achieve the threshold, use the complex SVM to recognition the region of the image.

Step5: Output the image recognition result N.

End

Through this algorithm, the image is classified by the classifier obtained in Algorithm 1, and iterative processing is performed to obtain the image recognition result.

\section{EXPERIMENT}

- Multiple Objects Recognition Based on CIFAR10 Dataset

The CIFAR-10 [14] is labeled subsets of the 80 million tiny images dataset. They were collected by Alex Krizhevsky, Vinod Nair, and Geoffrey Hinton. The CIFAR-10 dataset consists of $60,00032 \times 32$ color images in 10 classes, with 6,000 images per class. There were 50,000 training images and 10,000 test images. The dataset is divided into five training batches and one test batch, each with 10,000 images. The test batch contains exactly 1,000 randomly-selected images from each class. The training batches contain the remaining images in random order, but some training batches may contain more images from one class than another. Between them, the training batches contain exactly 5,000 images from each class. The original dataset is a single target image. In order to verify the accuracy of the three-way decision classifiers, four images in the original dataset are synthesized in this experiment, each test item has four targets to be recognized.

The experimental results are shown in Tab 2, this experiment uses the 4,000 pictures provided in the dataset, test each type of random selection of 400 pictures, each experiment for 100 pictures, the final results take the average. As shown in the above table, the average accuracy rate of the method is $87.58 \%$ and the average F-Measure is $87.06 \%$ in the dataset CIFAR-10, and the recognition rate based on the three-way decision classifiers can get higher recognition rate and accuracy rate.

Table 2: Based on the three-way decision CIFAR-10 multi-target recognition rate.

\begin{tabular}{|c|c|c|c|c|c|c|c|c|c|c|}
\hline Species & $\begin{array}{c}\text { Airplan } \\
\mathrm{e}\end{array}$ & $\begin{array}{c}\text { Automobil } \\
\mathrm{e}\end{array}$ & Brid & Cat & Deer & Dog & Frog & Horse & Ship & Truck \\
\hline Quantity & 100 & 100 & 100 & 100 & 100 & 100 & 100 & 100 & 100 & 100 \\
\hline Precision & $86 \%$ & $86.5 \%$ & $90 \%$ & $83.75 \%$ & $81.75 \%$ & $86 \%$ & $89.5 \%$ & $90.5 \%$ & $90 \%$ & $91.75 \%$ \\
\hline Recall & $91.68 \%$ & $89.48 \%$ & $92.08 \%$ & $87.22 \%$ & $84.61 \%$ & $88.64 \%$ & $81.55 \%$ & $92.47 \%$ & $89.78 \%$ & $83.23 \%$ \\
\hline F-Measure & $88.75 \%$ & $87.96 \%$ & $91.03 \%$ & $85.43 \%$ & $83.16 \%$ & $85.99 \%$ & $85.31 \%$ & $91.47 \%$ & $89.89 \%$ & $87.34 \%$ \\
\hline
\end{tabular}

- Multiple Objects Recognition Based on Car detection on UIUC Dataset

The UIUC dataset [15-19] captures images of side views of cars with resolution $40 \times 100$ pixels. The training set contains 550 positive samples and 500 negative samples. The test set is divide into two subsets: 170 single-scale test images, containing 200 cars at roughly the same scale as in the training set, and 108 multi-scale test images, containing 139 cars at various scales.

Table 3: Recognition rate comparison based on UIUC dataset.

\begin{tabular}{|l|c|c|c|}
\hline \multirow{2}{*}{ Method } & \multicolumn{3}{|c|}{ UIUC Dataset } \\
\cline { 2 - 4 } & Precision & Recall & F-Measure \\
\hline
\end{tabular}

\begin{tabular}{|c|c|c|c|}
\hline CS-AdaBoost [15] & $95.5 \%$ & $95.1 \%$ & $95.3 \%$ \\
\hline AdaBoost+LDA [16] & $97.8 \%$ & $99.41 \%$ & $98.6 \%$ \\
\hline AdaBoost [17] & $98.6 \%$ & $98.6 \%$ & $98.6 \%$ \\
\hline Pruning [18] & $97.8 \%$ & $99.41 \%$ & $98.6 \%$ \\
\hline FD [19] & $99.28 \%$ & $97.8 \%$ & $98.6 \%$ \\
\hline 3WD & $99.41 \%$ & $99.12 \%$ & $99.26 \%$ \\
\hline
\end{tabular}

The experimental results are shown in Table 3, for the UIUC dataset, where the dataset in the image characteristics of which 3WD (three-way decision) for the proposed method, FD (fast detection framework) for the fast detection framework [19], all the data in the table is average, Figure 4 shown that the accuracy of image recognition based on three-way decision is higher than 
that of the fast detection framework (FD). The method of recognition rate of $99.41 \%$, F-Measure of $99.26 \%$ slightly higher than the FD method.

- Multiple Objects Recognition Based on Car detection on KITTI Dataset

The KITTI dataset [19] was co-founded by the Karlsruhe Institute of Technology and the Toyota American Technology Research Institute. It is the largest computerized visual algorithm evaluation data set in the world. The dataset is a recently proposed challenging dataset which consists of 7481 training images and 7518 test images, comprising more than 80 thousand of annotated objects in traffic scenes. In this paper, the image of the car is used to experiment and compare, and the method of comparison is also used as the experimental object of the car information in the image. The experimental results as shown in Tab 4.

Table 4: Recognition rate comparison based on KITTI dataset.

\begin{tabular}{|c|c|c|c|}
\hline \multirow{2}{*}{ Method } & \multicolumn{3}{|c|}{ KITTI Dataset } \\
\cline { 2 - 4 } & $\begin{array}{c}\text { Precisio } \\
\mathrm{n}\end{array}$ & Recall & F-Measure \\
\hline mBoW [20] & $36.02 \%$ & $56.3 \%$ & $43.93 \%$ \\
\hline MDPM [21] & $71.19 \%$ & $65.05 \%$ & $67.98 \%$ \\
\hline DPM- & $74.33 \%$ & $69.92 \%$ & $72.06 \%$ \\
C8B1[22] & & & \\
\hline OC-DPM [23] & $74.94 \%$ & $69.53 \%$ & $72.13 \%$ \\
\hline AOG [24] & $80.26 \%$ & $74.23 \%$ & $77.13 \%$ \\
\hline SubCat [25] & $81.94 \%$ & $76.98 \%$ & $79.38 \%$ \\
\hline Regionlets & $84.75 \%$ & $78.83 \%$ & $81.68 \%$ \\
\hline [9,10] & & & \\
\hline FD [18] & $87.19 \%$ & $80.85 \%$ & $83.9 \%$ \\
\hline 3WD & $89.27 \%$ & $88.45 \%$ & $88.86 \%$ \\
\hline
\end{tabular}

As shown in Tab 4, the recognition rate of the image recognition method (3WD) based on three-way decision is $89.27 \%$, the F-Measure is $88.86 \%$, higher than the fast frame method (FD). The methods used in this paper have higher accuracy and F-Measure than other methods.

\section{CONCLUSION}

In this paper, we proposed a novel method image recognition that based on three-way decision theory. firstly, we invoked the three-way decision method in the field of image recognition. Secondly, we improved the composite SVM algorithm that through the various of classifier. Finally, through these two methods, the image recognition rate is improved. The experimental results shown that in the case of the same experimental dataset, the image recognition based on three-way decision has a significant improvement in accuracy compared with the traditional image recognition method. To the future work, Optimization of the running time of image recognition is an important topic. And the same time, we will attempt on improve the image recognition method based on three-way decision classifier, and use a variety of recognition methods for image recognition to further improve the image recognition accuracy.

\section{ACKNOWLEDGEMENTS}

This work is supported by the National Natural Science Foundation of China (NSFC) under Grant (no. 61533020, 61309014); Major Science and Technology Project for "Innovation of Common Key Technology in Key Industries " of CQ under Grant (no. cstc2017zdcyzdzx0046); Natural Science Foundation Project of CQ CSTC under Grant (no. cstc2017jcyjAX0408).

\section{REFERENCES}

1. Smith, J., 1998. The book, The publishing company. London, $2^{\text {nd }}$ edition.

2. Xuelong Li, Zhengkai Liu. An Efficient Semantic Image Classification Method [J]. Journal of Circuits and Systems, 2002, 7(2):22-25.

3. Hao Zhang. Extraction and Analysis of Semantic Image Features[D]. Shanghai Jiao Tong University, 2006.

4. Yao Y. An Outline of a Theory of Three-Way Decisions[M]// Rough Sets and Current Trends in Computing. Springer Berlin Heidelberg, 2012:1-17.

5. Dun Liu. The three-way decision and granular computing[M]. China Science Pubic, 2013.

6. Liang Decui. The research of decision-making method based on decision-theoretic rough set under the fuzzy environment[D]. Southwest Jiaotong University, 2014.

7. Li Deyi, Liu Changyu, Du Yi, et al. Artificial Intelligence with Uncertainty[J]. Journal of Software, 2004, 15(11):1583-1594.

8. Wang $\mathrm{X}$, Yang $\mathrm{M}$, Zhu S, et al. Regionlets for Generic Object Detection[C]// IEEE International Conference on Computer Vision. IEEE, 2014:17-24.

9. Long C, Wang X, Hua G, et al. Accurate Object Detection with Location Relaxation and Regionlets Re-localization[C]// Asian Conference on Computer Vision. Springer, Cham, 2014:260-275.

10. Shao Xiaoyan, Li Yan, Li Lihong. Transformation of no-commitment decision of three-way decision based on the maximization of income[J]. Microcomputer its Applications, 2017, 36(8):79-82.

11. Yin Ye, Ke Deying, Liu Chuanyong. Throry of trinary decision and its application[J]. Journal of Shanghai Normal University (Natural Sciences), 2015, 44(1):95-104.

12. Liu Baoxiang, Li Yan, Sun Jie. Three-way decisions and related theoretical research[J]. Microcomputer its Applications, 2014(12):1-3.

13. Carvalho E F, Engel P M. Convolutional Sparse Feature Descriptor for Object Recognition in CIFAR-10[C]// Brazilian Conference on Intelligent Systems. IEEE Computer Society, 2013:131-135.

14. Masnadishirazi H, Vasconcelos N. Cost-sensitive boosting.[J]. IEEE Transactions on Pattern Analysis \& Machine Intelligence, 2010, 33(2):294-309.

15. Wu J, Brubaker S C, Mullin M D, et al. Fast Asymmetric Learning for Cascade Face Detection[J]. 
IEEE Transactions on Pattern Analysis \& Machine Intelligence, 2008, 30(3):369.

16. Viola $\mathrm{P}$, Jones M. Robust real-time face detection[J]. International Journal of Computer Vision, 2004, 57(2):137-154.

17. Paisitkriangkrai S, Shen C, Hengel A V D. Asymmetric Pruning for Learning Cascade Detectors[J]. IEEE Transactions on Multimedia, 2014, 16(5):1254-1267.

18. $\mathrm{Hu} \mathrm{Q}$, Paisitkriangkrai S, Shen C, et al. Fast Detection of Multiple Objects in Traffic Scenes with a Common Detection Framework[J]. IEEE Transactions on Intelligent Transportation Systems, 2016, 17(4):1002-1014.

19. Behley J, Steinhage V, Cremers A B. Laser-based segment classification using a mixture of bag-ofwords[C]// Ieee/rsj International Conference on Intelligent Robots and Systems. IEEE, 2013:41954200 .

20. Forsyth D. Object Detection with Discriminatively Trained Part-Based Models[J]. IEEE Transactions on Pattern Analysis \& Machine Intelligence, 2014, 47(2):6-7.

21. Yebes J J, Bergasa L M, Arroyo R, et al. Supervised learning and evaluation of KITTI's cars detector with DPM[C]// Intelligent Vehicles Symposium Proceedings. IEEE, 2014:768-773.

22. Pepikj B, Stark M, Gehler P, et al. Occlusion Patterns for Object Class Detection[C]// Computer Vision and Pattern Recognition. IEEE, 2013:32863293.

23. $\mathrm{Li} \mathrm{B}, \mathrm{Wu} \mathrm{T}$, Zhu $\mathrm{S} \mathrm{C}$. Integrating Context and Occlusion for Car Detection by Hierarchical And-Or Model[M]// Computer Vision - ECCV 2014. Springer International Publishing, 2014:652-667.

24. Ohn-Bar E, Trivedi M M. Fast and Robust Object Detection Using Visual Subcategories[C]// Computer Vision and Pattern Recognition Workshops. IEEE, 2014:179-184. 